\title{
Real barchan dune collisions and ejections
}

\author{
Chris H. Hugenholtz ${ }^{1,2}$ and Thomas E. Barchyn ${ }^{1}$ \\ Received 9 November 2011; revised 19 December 2011; accepted 22 December 2011; published 26 January 2012.
}

[1] From high-resolution satellite imagery of barchan sand dunes, we provide geomorphological evidence of collisions that result in the ejection of a barchan from the wake of another barchan dune. Previous interpretations suggest this outcome is evidence of soliton or solitary wave behaviour; however, the physical mechanisms for mass exchange are not fully understood, resulting in debate. Our evidence and interpretation indicates that mass is transferred to the upwind barchan by shadowing a portion of downwind barchan's stoss slope. Turbulent, unsaturated airflow erodes the surface between the dunes, creating a smaller dune that ejects from the wake region. Previous observations lacked the spatial resolution required to document this process; therefore, our observations clarify the collision dynamics of barchans. A broader implication of our observations is the role of collisions in maintaining an "equilibrium" size distribution in barchan swarms. Citation: Hugenholtz, C. H., and T. E. Barchyn (2012), Real barchan dune collisions and ejections, Geophys. Res. Lett., 39, L02306, doi:10.1029/2011GL050299.

\section{Introduction}

[2] The barchan sand dune is the most pervasive dune type in areas with strong uni-directional wind and low sand supply. They typically propagate in groups or swarms stretching downwind from a source deposit. Within a swarm, barchans vary in size and mobility, with smaller barchans moving faster than larger ones. This heterogeneity creates a highly dynamic and interactive landscape characterized by collisions, form-modifications, and breeding of new barchans. Despite considerable progress in resolving the morphology and kinematics of barchans, our understanding of their dynamics and interactions is still limited by the long timescales required to resolve changes. To this end, flume experiments and numerical models have been used to supplement field observations [e.g., Schwämmle and Herrmann, 2003; Endo et al., 2004; Hersen et al., 2004; Durán et al., 2005; 2011; Hersen, 2005; Hersen and Douady, 2005; Katsuki et al., 2011]. These approaches are invaluable for providing insight and developing hypotheses; however, there are few real-world examples that have been used to verify and refine model and laboratory simulations. In some cases this has prompted debate as to the representativeness of model output [see Schwämmle and Herrmann, 2003; Durán et al., 2005; Hersen, 2005; Livingstone et al., 2005; Schwämmle and Herrmann, 2005].

\footnotetext{
${ }^{1}$ Department of Geography, University of Lethbridge, Lethbridge, Alberta, Canada.

${ }^{2}$ Faculty of Environmental Design, University of Calgary, Calgary, Alberta, Canada.

Copyright 2012 by the American Geophysical Union. 0094-8276/12/2011GL050299
}

[3] The collision of two barchans can produce a number of outcomes (see review by Kocurek et al. [2010]), which is related to the dune-size ratio and the alignment or offset of their downwind axes. Despite the profusion of different terms to describe the processes (e.g., breeding, budding, merging, absorbing, coalescing, splitting, reorganization, spawning, emission, ejection, solitary wave behaviour, etc.), there is some consistency in the types of outcomes derived from numerical models and flume experiments. When the dune-size ratio is large, collisions typically result in the smaller, upwind ('impacting') barchan being absorbed by the larger, downwind ('impacted') barchan. As the ratio decreases the number of possible outcomes increases, ranging from the creation of one of more barchan dunes along the arms of the downwind dune, to ejections of dunes from the slipface [Schwämmle and Herrmann, 2003; Endo et al., 2004; Durán et al., 2005; Hersen, 2005; Hersen and Douady, 2005; Kocurek et al., 2010; Katsuki et al., 2011]. A similar range of outcomes is produced by changing the dune alignment.

[4] Understanding the outcome of collisions between barchans is important for modeling the long term trends of barchan dune fields and resulting sedimentary record [Lee et al., 2005; Diniega et al., 2010; Durán et al., 2011]. If interactions are dominantly constructive (e.g., coalescence), dunes can increase in size to eventually form mega-dunes. If some interactions are destructive (e.g., ejections), dunes could maintain an 'equilibrium' size distribution [e.g., Durán et al., 2011].

[5] The most controversial outcome of barchan dune collision is the soliton or solitary wave behaviour [Schwämmle and Herrmann, 2003], whereby dunes appear to cross through one another without being altered. The idea is that the upwind dune captures incoming sand, thereby depriving the downwind dune of sand, which responds by shrinking and accelerating downwind [Durán et al., 2005; Vermeesch, 2011]. It "appears" as though the former passes through the latter when in fact the colliding dunes merely change in size and speed due to changes in their respective volumes. Although this idea has been challenged owing to a lack of geomorphological evidence [Livingstone et al., 2005], there is mounting evidence showing that collisions can produce smaller dunes in the wake region of another dune [see Ewing and Kocurek, 2010, Figure 7; Vermeesch, 2011], which appears to support the original explanation proposed by Schwämmle and Herrmann [2003].

[6] Vermeesch [2011] recently documented soliton-like behaviour with multi-temporal satellite imagery of dunes in the Bodélé Depression in northern Chad. In this study, we present new, independently-derived and complimentary observations of barchan collisions in Namibia with highresolution imagery. We present a detailed interpretation of processes during the collision-ejection sequence that is consistent with dune airflow research. Key form modifications 


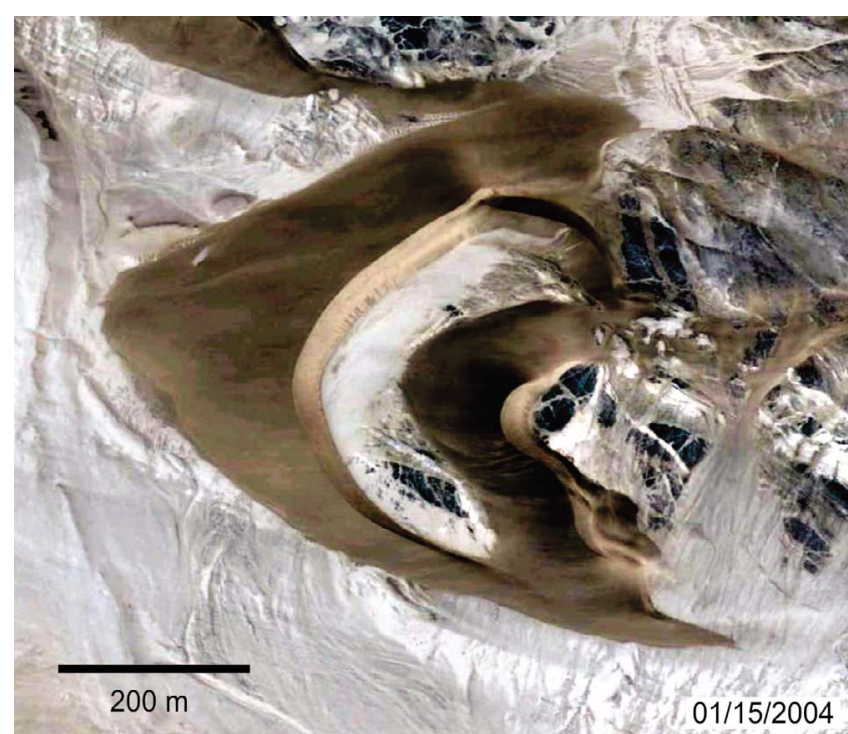

Figure 1. Example of a small barchan located in the wake of a larger barchan $\left(26^{\circ} 52^{\prime} 40.63^{\prime \prime} \mathrm{S}, 15^{\circ} 19^{\prime} 53.04^{\prime \prime} \mathrm{E}\right.$; image obtained from Google ${ }^{\mathrm{TM}}$ Earth). Google Earth imagery (C) Google Inc. Used with permission.

indicate that the colliding dunes coalesce while a portion of the downwind barchan is eroded by turbulent airflow in the lee of the upwind barchan, creating a smaller dune that ejects from the wake region. Thus, while collisions might resemble solitons, in that a smaller dune is present both before and after collision, the physical process is related to airflow perturbations and mass absorption.

\section{Study Site and Methods}

[7] We present examples from two sites along the coastal plain of Namibia, near the towns of Luderitz and Walvis Bay. To study their dynamics we used high-resolution, multi-date images from Google ${ }^{\mathrm{TM}}$ Earth. The images were obtained from the QuickBird and GeoEye-1 satellites. The positional accuracy of these images is unknown; however, because our focus is to document the morphological response of barchans to collisions, our observations and interpretations are minimally-influenced by positional error. To improve the co-registration of multi-date image sequences we rectified the images by using the earliest image at each site as the reference for all subsequent images. This ensured that dune morphological changes and displacements were geometrically-referenced to the earliest image.

\section{Results and Discussion}

[8] All along the coastal plain of Namibia we identified numerous examples of small barchans located within the wake region of larger barchans (Figures 1 and S1). ${ }^{1}$ In many instances the smaller barchans were connected to the slipfaces of the larger barchans by wings extending upwind from the horns of the former. Image pairs e-f and $g$-h in Figure S1 show the ejection of smaller barchans downwind of larger barchans. According to previous explanations, the smaller dunes can be interpreted in two ways: (i) as evidence favouring the soliton analogy, whereby the dune shape, i.e. the soliton, is somehow transferred "through" the other [Schwämmle and Herrmann, 2003, 2005; Durán et al., 2005; Vermeesch, 2011]; and (ii) that the smaller barchan calved from one of the horns and migrated laterally into the wake of the larger barchan due to cross-winds [Livingstone et al., 2005]. However, if cross-winds were a causal mechanism we would expect to find distinct form modifications across the entire dunefield, including "occluded" or "drop" morphologies [see Parteli et al., 2009], which we do not. Thus, while we can tentatively confirm the cross-wind scenario is not necessary to explain this configuration, we can neither confirm nor deny the soliton scenario from the single-date imagery.

[9] In Figure 2 we show an example of a collision and the formation of a barchan attached by wings to a larger barchan. This example further indicates the cross-wind scenario is not a requisite for a barchan to form within the wake region. The start of the sequence shows two barchans approaching the stoss slope of a larger barchan that is laterally-linked with two other large barchans (Figure 2a). When they collide, the impacting barchans coalesce on or near the base of the stoss slope of the impacted barchan (Figure $2 \mathrm{~b}$ ). The final image shows a smaller barchan emerging from within the wake region of a larger barchan (Figure 2c). The former is still linked to the latter by wings, but we expect it eventually detaches and migrates downwind (see Figures S1e-S1h). Endo et al. [2004] also noted short wings connecting to the larger barchan before the smaller barchan was fully ejected.

[10] In Figure 2d we show the positions of dune brinklines digitized from images in $2 \mathrm{a}-\mathrm{c}$ and the corresponding

\footnotetext{
${ }^{1}$ Auxiliary materials are available in the HTML. doi:10.1029/ 2011 GL050299.
}

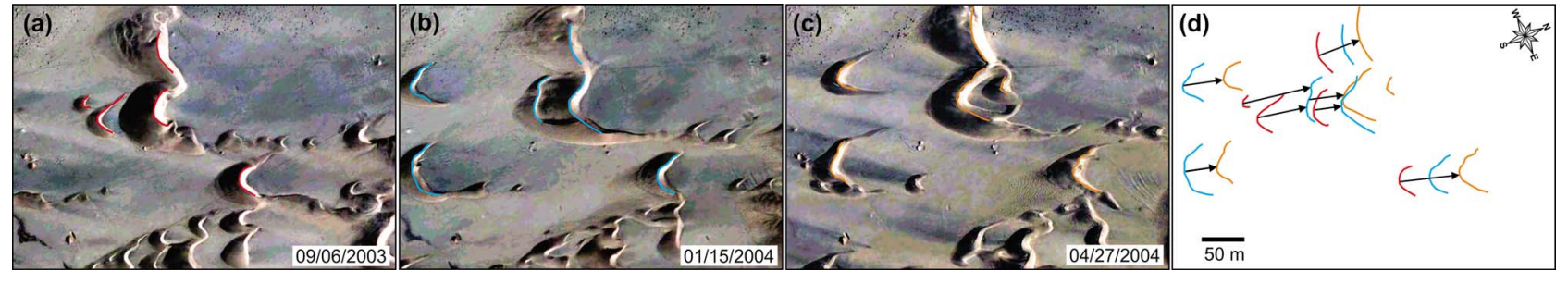

Figure 2. (a-c) Barchan collision-ejection sequence ( $\left.26^{\circ} 51^{\prime} 45.25^{\prime \prime} \mathrm{S}, 15^{\circ} 14^{\prime} 18.65^{\prime \prime} \mathrm{E}\right)$. Image dates shown in bottom right corner (QuickBird images obtained from Google ${ }^{\mathrm{TM}}$ Earth). Google Earth imagery (C) Google Inc. Used with permission. (d) Brink line positions, with colors corresponding to each image: red (09/06/2003), blue (01/15/2004), orange (04/27/ 2004). Arrows denote relative displacement vectors for selected brink lines. 

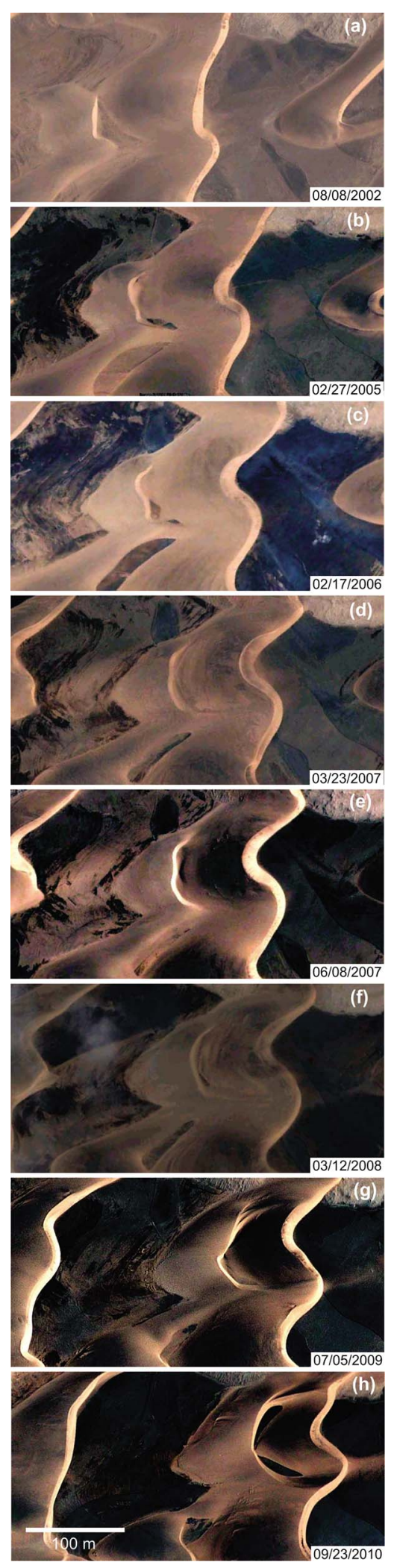

Figure 3. $(\mathrm{a}-\mathrm{h})$ Barchan collision-ejection sequence $\left(22^{\circ} 59^{\prime} 43.38^{\prime \prime} \mathrm{S}, 14^{\circ} 28^{\prime} 39.99^{\prime \prime} \mathrm{E}\right)$. Figures 3a-3f are from QuickBird, while Figures $3 \mathrm{~g}-3 \mathrm{~h}$ are from GeoEye-1. Image dates shown in bottom right corner (images obtained from Google ${ }^{\mathrm{TM}}$ Earth). Google Earth imagery (C) Google Inc. Used with permission. displacement vectors. The brinklines allows us to track individual dunes through the image sequence. We also present brinkline positions and vectors of adjacent dunes for context. Figure 2d shows that instead of displacing downwind, the brinkline of the larger dune in the second and third images (Figures $2 b$ and $2 c$ ) shifts slightly to the west. To our knowledge, there is no physical mechanism that could explain this direction of displacement given the northward displacement of adjacent dunes. As denoted by the vectors, what is more probable is that the upwind dune migrated onto the stoss of the downwind dune, burying a portion of the latter and eventually forming the larger dune in Figure 2c, which represents the outcome of the coalescence. Thus, the brinkline of the larger barchan in Figure 2c corresponds to the brinkline of the upwind barchan in Figure $2 b$.

[11] A more detailed example of the collision and ejection sequence at Walvis Bay is provided in Figure 3. In this example the dune-size ratio appears to be smaller than in Figure 2. The sequence shows a similar chronology, whereby the upwind dune coalesces along the lower stoss slope of the downwind dune (Figures 3a-3e) before eroding the latter, forming a smaller dune in its wake attached by wings. The elongation of the upwind dune's horns in Figures $3 \mathrm{e}-3 \mathrm{~h}$ appears to establish a lateral boundary for the formation of the ejected dune.

[12] Our interpretation of the processes responsible for the sequences in Figures 2 and 3 is consistent with previous explanations derived from flume experiments [Endo et al., 2004; Hersen and Douady, 2005]. The process sequence is illustrated in Figure 4. As the upwind barchan approaches the stoss slope of the downwind barchan, it transfers sediment outflux from its horns directly to the stoss of the latter (Figures $3 \mathrm{a}$ and $3 \mathrm{~b}$ ). This begins to modify the upwind sediment influx. The center of the downwind barchan's stoss slope also becomes sand-deprived because it is blocked from receiving sand influx by the upwind barchan's separation cell (i.e., lee eddy). The separation cell is included in the wake region [see McLean and Smith, 1986]. As the upwind barchan approaches the stoss of the downwind barchan the reduction in shear stress in the separation cell begins to shelter a portion of the downwind dune's stoss slope. The upwind dune overrides this stationary sediment along the lower stoss of the downwind dune, absorbing it and increasing in size. At this stage the separation cell contracts, which is consistent with wind tunnel and field measurements of closely-spaced dunes [Walker and Nickling, 2003; Baddock et al., 2011]. In Figure 4c the horns of the upwind dune elongate as it begins coalescing with the downwind dune, while erosion takes place along the crest where turbulent airflow reaching the surface is unsaturated, which means it has residual capacity to entrain sand, causing erosion. This may be further compounded by increased turbulence and shear stress on the stoss of a downwind dune [Ferreira and Fino, 2012; Palmer et al., 2012], which may enhance erosion [see also Endo et al., 2004; Hersen and Douady, 2005; Kocurek et al., 2010], especially beyond the point of flow reattachment where the internal boundary layer redevelops [McLean and Smith, 1986; Walker and Nickling, 2003]. Presumably, the pattern of erosion on the downwind barchan denotes the pattern of airflow recovery. Continued erosion carves out a smaller barchan that is connected to the larger dune by wings extending back from the horns (Figure 4d). Finally, the smaller, downwind dune 


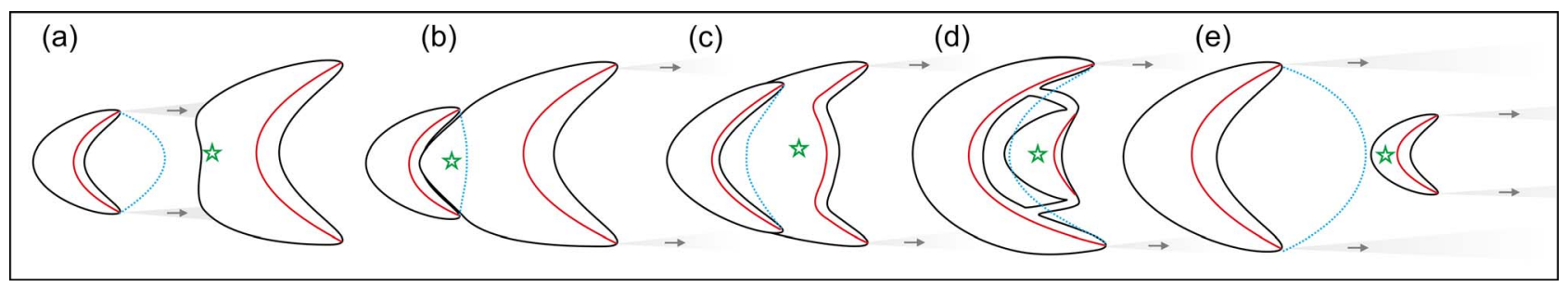

Figure 4. (a-e) Illustrated interpretation of the collision-ejection sequence. Blue lines correspond to the assumed edge of the separation cell, red lines correspond to brink lines, and stars correspond to the description given in the text. The dashed line in Figure 4c denotes an erosional boundary.

ejects and decreases in size due to sheltering until it disappears or survives long enough to impact with another dune downwind (Figure 4e; see also Figure S1h).

[13] As the downwind dune pulls away from the upwind dune, it is sheltered from sediment influx from upwind and increases in speed once it clears the separation cell because it is losing sediment and decreasing in size. This effect may further promote dune separation. However, in closelyspaced dunes, the whole process may repeat itself once the ejected dune encounters another downwind dune (e.g., Figures S1g and S1h), or conversely, it may disappear if dunes are wider-spaced.

[14] The transfer of mass from the downwind dune to the upwind dune is worthwhile to clarify. First, the upwind dune shadows the sediment that reaches the downwind dune, further promoting a change in size between the two dunes. This process is plausible and widely endorsed as a cause of "soliton" behaviour [Vermeesch, 2011]. However, similar morphology observed in a flume by Endo et al. [2004] occurs in the absence of sediment influx, suggesting sediment sheltering is not a requisite for barchan ejections. Second, as the upwind dune overrides the stoss of the downwind dune, its wake region reduces near surface shear stress, limiting the sediment in this area from being transported downwind. As the dunes are both migrating downwind during the collision, stationary sediment is absorbed into the upwind dune [see Diniega et al., 2010, Figure 3]. This process helps explain why the size ratio of the colliding dunes matters. A large ratio increases the closing speed of the two dunes, reducing the amount of time the upwind dune is in a position to shelter the stoss of the downwind dune. Thus, a small upwind dune is less likely to grow sufficiently via sediment absorption to outsize the downwind dune and result in an ejection. Conversely, a smaller ratio increases the amount of time that sediment can be absorbed into the upwind dune as the two dune speeds are similar (Figure 3). From our interpretation, this is the dominant process for mass transfer from the downwind to upwind dune.

[15] Our observations suggest that the shape of the newlydeveloped barchan along the slipface of the larger barchan is an erosional by-product of the lee-side airflow pattern. The process is similar to bed form repulsion [Kocurek et al., 2010], where the impacting barchan modifies both the airflow and sediment supply reaching the impacted barchan, which then erodes and ejects a new dune, while the remnants of the original dunes coalesce. Simulation-based explanations highlighting soliton behaviour have thus far underemphasized the role of lee-side airflow during collisions. Part of this distinction may relate to the way numerical and analytical dune models characterize airflow in the wake. In the models airflow in the wake is represented by simplified expressions (i.e., ellipse, polynomial, shadow zone) involving idealized flow conditions in this region. While these simplified expressions are needed in the absence of empirical evidence, they may misrepresent conditions that cause sand transport in this region, while also yielding abstract explanations of the collision-ejection process.

[16] We note that much of this debate [Livingstone et al., 2005; Schwämmle and Herrmann, 2005; Vermeesch, 2011] may be exacerbated by treatment of barchan dunes as discrete objects. Instead of being contiguous objects, dunes are simply collections of particles (sand). Mid-collision, barchan dunes change into a coalesced form that is not easily described as a discrete barchan. Thus, debate of whether the two dunes i) pass through each other, ii) touch each other and separate, or iii) coalesce and subsequently separate, could be largely semantic.

[17] In conjunction with Vermeesch [2011], our observations highlight the geomorphic consequence of dune collisions in dunefields. Collisions resulting in smaller or similarsized dunes are important in regulating the mean dune size. If interactions are primarily constructive, dunes increase in size, potentially leading to the formation of mega-dunes; however, if interactions are deconstructive, the mean dune size remains small. Some researchers have simulated dunefields by representing dunes by points or lines and invoking generalized interaction functions (based on modeled ejections) to iterate forward in time [Lee et al., 2005; Diniega et al., 2010; Durán et al., 2011]. These efforts highlight the importance of deconstructional events such as ejections in limiting runaway dune coalescence and raise significant questions about the boundary conditions responsible for mega-dune formation.

\section{Conclusions}

[18] Through multi-temporal satellite imagery we have presented geomorphological evidence indicating that collisions between barchans can result in the ejection of a new barchan from within the wake region of another barchan. The mechanism we propose to account for this outcome involves mass transfer through sheltering and absorption of sediment by the upwind dune coupled with turbulent, unsaturated airflow that erodes a gap between the dunes. Our observations and interpretations confirm the main sequences of the collision-ejection process noted in flume experiments, simulations and satellite imagery [Endo et al., 2004; Katsuki et al., 2011; Vermeesch, 2011], and highlight the role of dune-dune interactions in regulating the dune-size distribution of barchan dune fields. 
[19] Acknowledgments. Financial support for this project was provided by NSERC and Alberta Innovates awards to $\mathrm{CHH}$. We thank Pieter Vermeesch and three anonymous reviewers for helpful comments.

[20] The Editor thanks Pieter Vermeesch and two anonymous reviewers for their assistance in evaluating this paper.

\section{References}

Baddock, M. C., G. F. S. Wiggs, and I. Livingstone (2011), A field study of mean and turbulent flow characteristics upwind, over and downwind of barchan dunes, Earth Surf. Processes Landforms, 36, 1435-1448, doi:10.1002/esp. 2161.

Diniega, S., K. Glasner, and S. Byrne (2010), Long-time evolution of models of aeolian dune fields: Influence of dune formation and collision, Geomorphology, 121, 55-68, doi:10.1016/j.geomorph.2009.02.010.

Durán, O., V. Schwämmle, and H. J. Herrmann (2005), Breeding and solitary wave behavior of dunes, Phys. Rev. E, 72, 021308, doi:10.1103/ PhysRevE.72.021308.

Durán, O., V. Schwämmle, P. G. Lind, and H. J. Herrmann (2011), Size distribution and structure of barchan dune fields, Nonlinear Processes Geophys., 18, 455-467, doi:10.5194/npg-18-455-2011.

Endo, N., K. Taniguchi, and A. Katsuki (2004), Observation of the whole process of interaction between barchans by flume experiments, Geophys. Res. Lett., 31, L12503, doi:10.1029/2004GL020168.

Ewing, R. C., and G. A. Kocurek (2010), Aeolian dune interactions and dune-field pattern formation: White Sands Dune Field, New Mexico, Sedimentology, 57, 1199-1219, doi:10.1111/j.1365-3091.2009.01143.x.

Ferreira, A. D., and M. R. M. Fino (2012), A wind tunnel study of wind erosion and profile reshaping of transverse sand piles in tandem, Geomorphology, doi:10.1016/j.geomorph.2011.10.024, in press.

Hersen, P. (2005), Flow effects on the morphology and dynamics of aeolian and subaqueous barchan dunes, J. Geophys. Res., 110, F04S07, doi:10.1029/2004JF000185.

Hersen, P., and S. Douady (2005), Collision of barchan dunes as a mechanism of size regulation, Geophys. Res. Lett., 32, L21403, doi:10.1029/ 2005GL024179.

Hersen, P., K. H. Andersen, H. Elbelrhiti, B. Andreotti, P. Claudin, and S. Douady (2004), Corridors of barchan dunes: Stability and size selection, Phys. Rev. E, 69, 011304, doi:10.1103/PhysRevE.69.011304.
Katsuki, A., M. Kikuchu, H. Nishimori, N. Endo, and K. Taniguchi (2011), Cellular model for sand dunes with saltation, avalanche and strong erosion: collisional simulation of barchans, Earth Surf. Processes Landforms, 36, 372-382, doi:10.1002/esp.2049.

Kocurek, G. A., R. C. Ewing, and D. Mohrig (2010), How do bedform patterns arise? New views on the role of bedform interactions within a set of boundary conditions, Earth Surf. Processes Landforms, 35, 51-63, doi:10.1002/esp.1913.

Lee, J. H., A. O. Sousa, E. J. R. Parteli, and H. J. Herrmann (2005), Modelling formation and evolution of transverse dune-fields, Int. J. Mod. Phys. C, 16, 1879-1892, doi:10.1142/S0129183105008400.

Livingstone, I., G. F. S. Wiggs, and M. C. Baddock (2005), Barchan dunes: Why they cannot be treated as 'solitons' or 'solitary waves,' Earth Surf. Processes Landforms, 30, 255-257, doi:10.1002/esp.1206.

McLean, S. R., and J. D. Smith (1986), A model for flow over twodimensional bed forms, J. Hydraul. Eng., 112, 300-317, doi:10.1061/ (ASCE)0733-9429(1986)112:4(300).

Palmer, J. A., R. Meja-Alvarez, J. L. Best, and K. T. Christensen (2012), Particle-image velocimetry measurements of flow over interacting barchans dunes, Exp. Fluids, doi:10.1007/s00348-011-1104-4, in press.

Parteli, E. J. R., O. Durán, H. Tsoar, V. Schwämmle, and H. J. Herrmann (2009), Dune formation under bimodal winds, Proc. Natl. Acad. Sci. U. S. A., 106, 22,085-22,089, doi:10.1073/pnas.0808646106.

Schwämmle, V., and H. J. Herrmann (2003), Solitary wave behaviour of sand dunes, Nature, 426, 619-620, doi:10.1038/426619a.

Schwämmle, V., and H. J. Herrmann (2005), Reply to the discussion on "Barchan dunes: Why they cannot be treated as "solitons" or "solitary waves," Earth Surf. Processes Landforms, 30, 517, doi:10.1002/ esp. 1228 .

Vermeesch, P. (2011), Solitary wave behavior in sand dunes observed from space, Geophys. Res. Lett., 38, L22402, doi:10.1029/2011GL049610.

Walker, I. J., and W. G. Nickling (2003), Simulation and measurement of surface shear stress over isolated and closely spaced transverse dunes in a wind tunnel, Earth Surf. Processes Landforms, 28, 1111-1124, doi:10.1002/esp.520.

T. E. Barchyn and C. H. Hugenholtz, Department of Geography, University of Lethbridge, 4401 University Dr., Lethbridge, AB T1K 3M4, Canada. (chris.hugenholtz@uleth.ca) 
Reproduced with permission of the copyright owner. Further reproduction prohibited without permission. 